\title{
Doppler ultrasound venous mapping of the lower limbs
}

\author{
Aldo Innocente Galeandro' \\ Giovanni Quistelli² \\ Pietro Scicchitano ${ }^{2}$ \\ Michele Gesualdo² \\ Annapaola Zito ${ }^{2}$ \\ Paola Caputo ${ }^{2}$ \\ Rosa Carbonara ${ }^{2}$ \\ Giuseppe Galgano 3 \\ Francesco Ciciarello ${ }^{4}$ \\ Sandro Mandolesi ${ }^{4}$ \\ Claude Franceschi ${ }^{5}$ \\ Marco Matteo Ciccone ${ }^{2}$ \\ 'Centro Diagnostica Globale and \\ ASL-TA, Taranto, Italy; ${ }^{2}$ Cardiovascular \\ Diseases Section, Department of \\ Emergency and Organ Transplantation \\ (DETO), University of Bari, Bari, \\ Italy; ${ }^{3}$ Cardiovascular Diseases \\ Section, Ente Ecclesiastico Ospedale \\ Generale Regionale F Miulli, Acquaviva \\ delle Fonti, Bari, Italy; ${ }^{4}$ Cardiology \\ Department, Policlinico Umberto I, \\ Sapienza University of Rome, Italy; \\ ${ }^{5}$ Vascular Laboratories of Hospitals \\ Saint Joseph and Pitié-Salpétrière, \\ Paris, France
}

This article was published in the following Dove Press journal:

Vascular Health and Risk Management

4 February 2012

Number of times this article has been viewed

Background: The study aim was to test the accuracy (intra and interobserver variability), sensitivity, and specificity of a simplified noninvasive ultrasound methodology for mapping superficial and deep veins of the lower limbs.

Methods: 62 consecutive patients, aged $62 \pm 11$ years, were enrolled. All underwent US-examinations, performed by two different investigators, of both legs, four anatomical parts, and 17 veins, to assess the interobserver variability of evaluation of superficial and deep veins of the lower limbs.

Results: Overall the agreement between the second versus the first operator was very high in detecting reflux (sensitivity 97.9, specificity 99.7, accuracy 99.5; $P=0.80$ at McNemar test). The higher CEAP classification stages were significantly associated with reflux (odds ratio: $1.778,95 \%$ confidence interval: 1.552-2.038; $P<0.001$ ) as well as with thrombosis (odds ratio: 2.765 , 95\% confidence interval: $1.741-4.389 ; P<0.001)$. Thus, our findings show a strict association between the symptoms of venous disorders and ultrasound evaluation results for thrombosis or reflux.

Conclusion: This study demonstrated that our venous mapping protocol is a reliable method showing a very low interobserver variability, which makes it accurate and reproducible for the assessment of the morphofunctional status of the lower limb veins.

Keywords: venous mapping, new methodology, sensitivity, specificity, accuracy

\section{Introduction}

In all Western world societies, chronic venous disease prevalence varies greatly from $2 \%-50 \%$ both in men and women. The broad range is due to variability in populations examined (age, gender, race, methods, and even definition of the same disease). ${ }^{1}$

This disease carries a large economic burden, since about 110,000 venous stripping procedures are performed per year in US, during the process of which 3 million working days are lost. ${ }^{2}$

The diagnosis and treatment of chronic venous diseases has undergone a quantum leap forward in recent decades, ${ }^{3}$ thanks to advances in understanding made possible by technical improvements in ultrasound equipment, diagnostic techniques, ${ }^{4}$ and communication standards related to research in anatomy and pathophysiology. ${ }^{5}$ Currently, high definition echo color Doppler (HD-ECD) is considered the gold standard for diagnosis of the venous system. ${ }^{6}$

Venous mapping obtained by using HD-ECD provides an accurate graphic representation of the deep and superficial venous systems, ${ }^{7}$ and the performance of venous mapping is now considered essential for correct management of venous diseases. ${ }^{8}$
Correspondence: Marco Matteo Ciccone Cardiovascular Diseases Section, Department of Emergency and Organ Transplantation (DETO), University of Bari, piazza G Cesare, I I, 70124 - Bari, Italy Tel +39-080-547879|

Fax +39-080-5478796

Email ciccone@cardio.uniba.it 
In fact, such an evaluation leads to improved knowledge about the hemodynamics of the venous system. This is important in order to ameliorate the decision about further treatment of venous diseases, for example, in the case of evaluating the real necessity to strip the saphenous vein in case of its insufficiency.

The aim of our study was to evaluate the accuracy (intra and interobserver variability), sensitivity, and specificity of a novel noninvasive ultrasound methodology for mapping superficial and deep veins of the lower limbs.

\section{Materials and methods Subjects}

A total of 62 consecutive patients (12 males and 50 females), aged $62 \pm 11$ years attending the cardiovascular diseases section of the Emergency and Organ Transplant Department (DETO) of Bari University General Hospital, Italy, were enrolled in the study. Inclusion criteria were the presence of venous disease symptoms (stage C0-C6 according to the Clinical, Etiology, Anatomy, and Pathophysiology [CEAP] classification). ${ }^{9,10}$ Exclusion criteria were deep venous thrombosis, heart diseases (coronary heart disease, arrhythmia, heart failure), vascular brain diseases (stroke or a transient ischemic attack), peripheral obstructive artery disease (intermittent claudication, delayed or absent peripheral pulses), chronic liver disease, known kidney disease or any other chronic severe disease, pregnancy, and severe obesity (body mass index $\geq 35$ ).

All patients underwent ultrasound examinations, performed by two different investigators, of both legs, four anatomical parts, and 17 veins (the total number of evaluations made by each operator was 3224), to assess the interobserver variability of evaluation of superficial and deep veins of the lower limbs.

The study was approved by the Institutional Review Board of Bari University General Hospital, Italy, and carried out in accordance with the principles of the Helsinki Declaration; all patients gave written informed consent before entering the study.

\section{Investigators}

All HD-ECD examinations (interobserver study) were carried out by two observers with experience in venous ultrasonography. One ultrasonographer, AIG, was a well-experienced vascular surgeon specializing in angiology (hundreds Doppler/year); MMC was an associate professor in cardiology specializing in angiology, chief of the vascular laboratory of Bari University, Italy, author of several papers within the vascular field, personally performing color Doppler ultrasound evaluation of the patients afferent to his laboratory.

Prior to the study, the two observers established a consensus regarding the definition of the four anatomical parts for the ultrasonographic study. The sonographers carried out the US examinations blinded, one immediately after the other.

\section{Ultrasound examination}

This observational study was performed using multifrequency 7.5-10 MHz Doppler ultrasound probes (Sonos 1500B; Hewlett Packard, Palo Alto, CA). The room temperature was maintained between $18^{\circ} \mathrm{C}$ and $22^{\circ} \mathrm{C}$.

The HD-ECD examination was performed in accordance with the following protocol: ${ }^{11}$

1. In orthostatic position.

2. Examination of the superficial veins of the lower limbs, with the patient standing upright on a stool, and the limb under examination rotated outward.

3. In supine position, with the patient lying on a padded bed. Naturally, examination in the standing position was carried out in a non-weight-bearing manner in order to eliminate bias during the development of the procedure. The following parameters were assessed: ${ }^{12}$

- Venous size (anteroposterior diameter),

- Venous reflux (spontaneous or during maneuvers),

- Vein thrombosis (compression ultrasonography method), after performing the identification of four anatomical parts (two for the thigh and two for the calf) with the following topographic determinants:

- 1st part: from the midpoint of the thigh to the inguinal ligament,

- 2nd part: from the kneecap to the midpoint of the thigh,

- 3rd part: from the midpoint of the calf to the kneecap,

- 4th part: from the heel to the midpoint of the leg. The veins explored for each part were the:

\section{Ist part}

- common femoral vein

- femoral vein

- saphenofemoral junction

- great saphenous vein

- anterior accessory saphenous vein of the thigh

- posterior accessory saphenous vein of the thigh

- Giacomini vein

\section{2nd part}

- great saphenous vein

- Giacomini vein 
- Hunter perforating vein

- popliteal vein

- femoral vein

\section{3rd part}

- popliteal vein

- tibioperoneal trunk

- great saphenous vein

- small saphenous vein

- anterior accessory saphenous vein of the calf

- posterior accessory saphenous vein of the calf

- Boyd perforating vein

\section{4th part}

- great saphenous vein

- small saphenous vein

- Cockett perforating vein

- anterior accessory saphenous vein of the calf

- posterior accessory saphenous vein of the calf

- anterior tibial veins

- posterior tibial veins

The maneuvers used during the study were:

- The Valsalva maneuver, which elicits reflux in pathological conditions. The maneuver was performed by blowing for 30 seconds into a tube connected to a mercury manometer to develop a pressure of $40 \mathrm{mmHg}$ in the upper airways. ${ }^{13,14}$

- Proximal and distal manual compression: venous reflux is detected on release of the compression.

We considered 500 milliseconds as the cutoff value for reflux in the superficial veins, deep femoral veins, and deep calf veins; 1000 milliseconds as the reflux cutoff value for the femoropopliteal veins, while the outward flow in the perforating veins was considered abnormal at $>350$ milliseconds. ${ }^{15}$ An example of the new venous mapping is shown in Figure 1.

\section{Venous mapping}

All data obtained during the HD-ECD were reported on a special two-dimensional scheme representation (MAP - see Figure 1). The venous map is drawn up in two steps: firstly the venous anatomy is depicted (phase 1) and secondly, the direction of flow and the hemodynamics of the venous system is depicted (phase 2). The resulting map shows, in a grid divided into four parts, the superficial venous system and the deep venous system of each limb. As indicated before, during the procedure we evaluated in detail the veins considered, looking for focal problem areas and determining the diameters and the hemodynamics at different levels. The division into four

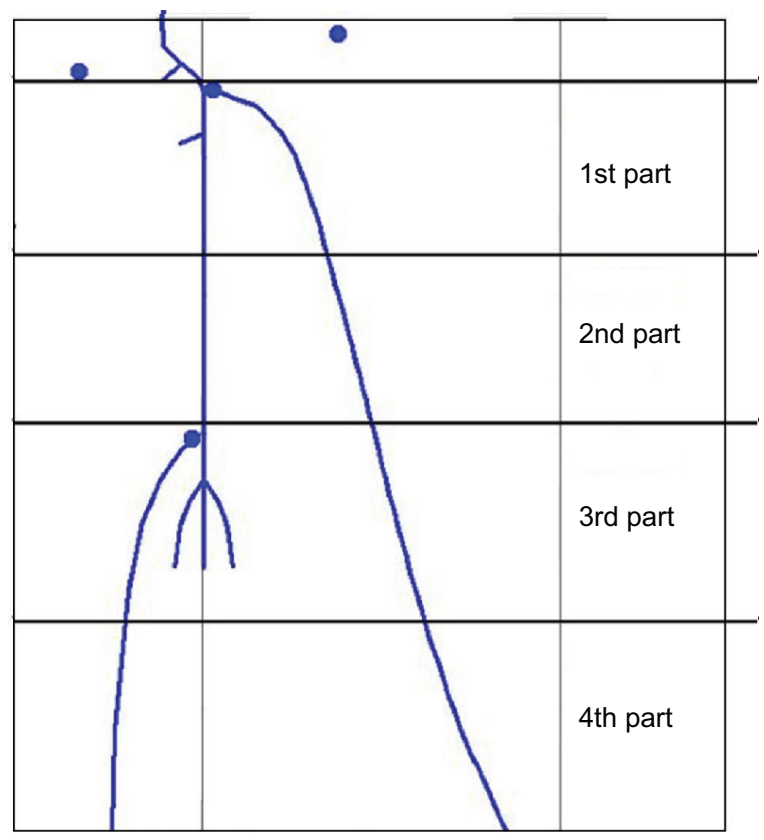

Figure I Scheme of the novel venous mapping.

parts leads us to enlarge the field of research, easily finding the zones to study because it is simple to divide the inferior leg into four zones rather than six subdivisions. The venous mapping remains equal to previous modalities of research but it is improved in detecting the areas of interest.

\section{Statistical analysis}

Continuous variables were expressed as mean \pm standard deviation and discrete variables as number and percentages. Agreement between the operators was tested by McNemar's test. The sensitivity, specificity, and accuracy were calculated for the second versus the first operator and expressed as a percentage. Data were analyzed using a mixed model with patients randomly fitted. The association between CEAP stage and reflux or thrombosis was estimated by means of odds ratios (ORs) and 95\% confidence intervals (CIs). Statistical analyses were performed with $\mathrm{R}$ statistical software (R Development Core Team, R Foundation for Statistical Computing, Vienna, Austria). A $P$ value $<0.05$ was considered significant.

\section{Results}

In this study, 124 legs were assessed in 62 patients, considering four anatomical parts of each lower limb, and 17 veins, for a total of 3224 evaluations made by each operator. Thromboses evaluated during the ultrasound evaluation were chronic with no acute ones detected during the evaluation of all the legs of the population sample. 
Table 1 shows the percentage of reflux and thrombosis observed in each of the four anatomical parts and in each vein explored along the entire course. Table 2 shows the percentage of reflux and thrombosis in each venous segment explored; it must be noted that that along their course, some veins may belong to more than one of the four parts.

The prevalence of reflux was negatively correlated with the anatomical part, showing lower values in the 2nd (OR: $0.636,95 \% \mathrm{CI}: 0.458-0.883 ; P=0.007$ ), 3rd (OR: $0.561,95 \%$ CI: $0.415-0.760 ; P<0.001$ ), and 4th part (OR: $0.476,95 \%$ CI: $0.349-0.651 ; P<0.001)$ than in the 1st (Figure 2A). Thrombosis was not associated with these parts (Figure 2B).

The 2nd investigator diagnosed 40 cases of saphenectomy in six patients and 26 cases of thrombosis in twelve patients (there was perfect agreement between the two operators in these evaluations of saphenectomy and thrombosis). In addition, 381 refluxing veins were diagnosed in 46 patients; in eight veins, the two observers were in disagreement as to the diagnosis of reflux. Thus, overall the agreement between the second versus the first operator was very high in detecting reflux (sensitivity 97.9, specificity 99.7, accuracy 99.5; $P=0.80$ at McNemar test).

Table I Percentage of reflux and thrombosis observed in each of four anatomical parts and in each vein explored along the entire course

\begin{tabular}{lll}
\hline Part & Reflux (\%) & Thrombosis (\%) \\
\hline Ist & 16.3 & 0.7 \\
2nd & 11.6 & 0.8 \\
3rd & 10.6 & 1.7 \\
4th & 9.3 & 0.0 \\
Veins & & \\
Anterior accessory saphenous & 16.9 & 1.6 \\
vein of the thigh & & \\
Anterior accessory saphenous & 10.9 & 0.0 \\
vein of the calf & & \\
Posterior accessory saphenous & 10.5 & 1.6 \\
vein of the thigh & & \\
Posterior accessory saphenous & 11.3 & 0.0 \\
vein of the calf & & \\
Saphenofemoral junction & 31.1 & 0.8 \\
Common femoral vein & 4.0 & 0.0 \\
Superficial femoral vein & 6.5 & 0.0 \\
Boyd perforating vein & 11.3 & 0.0 \\
Hunter perforating ring vein & 15.3 & 0.0 \\
Cockett perforating ring vein & 6.5 & 0.0 \\
Small saphenous vein & 2.4 & 0.0 \\
Great saphenous vein & 23.4 & 1.1 \\
Anterior tibial veins & 12.9 & 0.0 \\
Posterior tibial veins & 12.9 & 0.0 \\
Tibioperoneal trunk & 4.0 & 4.8 \\
Giacomini vein & 10.5 & 0.0 \\
\hline
\end{tabular}

Table 2 Percentage of reflux and thrombosis veins in the four anatomical parts

\begin{tabular}{|c|c|c|c|}
\hline Veins & Part & Reflux (\%) & Thrombosis (\%) \\
\hline $\begin{array}{l}\text { Anterior accessory } \\
\text { saphenous vein of the thigh }\end{array}$ & Ist & 16.9 & 1.6 \\
\hline $\begin{array}{l}\text { Anterior accessory } \\
\text { saphenous vein of the calf }\end{array}$ & $3 r d$ & 13.7 & 0.0 \\
\hline $\begin{array}{l}\text { Anterior accessory } \\
\text { saphenous vein of the calf }\end{array}$ & 4th & 8.1 & 0.0 \\
\hline $\begin{array}{l}\text { Posterior accessory } \\
\text { saphenous vein of the thigh }\end{array}$ & Ist & 10.5 & 1.6 \\
\hline $\begin{array}{l}\text { Posterior accessory } \\
\text { saphenous vein of the calf }\end{array}$ & $3 r d$ & 14.5 & 0.0 \\
\hline $\begin{array}{l}\text { Posterior accessory } \\
\text { saphenous vein of the thigh }\end{array}$ & 4th & 8.1 & 0.0 \\
\hline Saphenofemoral junction & Ist & 31.1 & 0.8 \\
\hline Common femoral vein & Ist & 4.0 & 0.0 \\
\hline Superficial femoral vein & Ist & 12.9 & 0.0 \\
\hline Superficial femoral vein & $2 n d$ & 0.0 & 0.0 \\
\hline Boyd perforating vein & $3 r d$ & 11.3 & 0.0 \\
\hline Hunter perforating vein & 2nd & 15.3 & 0.0 \\
\hline Popliteal vein & 2nd & 4.8 & 2.4 \\
\hline Cockett perforating vein & 4th & 6.5 & 0.0 \\
\hline Small saphenous vein & $3 r d$ & 2.4 & 0.0 \\
\hline Small saphenous vein & 4 th & 2.4 & 0.0 \\
\hline Great saphenous vein & Ist & 28.7 & 0.9 \\
\hline Great saphenous vein & 2nd & 29.6 & 1.7 \\
\hline Great saphenous vein & $3 r d$ & 20.9 & 1.7 \\
\hline Great saphenous vein & 4 th & 14.7 & 0.0 \\
\hline Anterior tibial veins & 4th & 12.9 & 0.0 \\
\hline Posterior tibial veins & 4th & 12.9 & 0.0 \\
\hline Tibioperoneal trunk & $3 r d$ & 4.0 & 4.8 \\
\hline Giacomini vein & Ist & 11.3 & 0.0 \\
\hline Giacomini vein & 2nd & 9.7 & 0.0 \\
\hline
\end{tabular}

Both operators were in agreement that 15 patients had no ultrasound findings of venous disease. The higher CEAP classification stages were significantly associated with reflux (OR: 1.778, 95\% CI: 1.552-2.038; $P<0.001$ ) (Figure 3 ) as well as with thrombosis (OR: $2.765,95 \%$ CI: $1.741-4.389$; $P<0.001)$. Thus, our findings show a strict association between the symptoms of venous disorders and ultrasound evaluation results for thrombosis or reflux.

\section{Discussion}

Chronic venous insufficiency and leg ulcers involving the lower limbs are estimated to affect approximately one-third of the Western population ${ }^{16}$ and $1-2$ people per 1000 in the general population, respectively. ${ }^{17}$ Clinical manifestations of chronic venous insufficiency of the lower limbs include purely symptomatic problems, varicosities, edema, and trophic skin lesions.

The CEAP classification is widely used internationally by venous disease specialists. It provides a precise description 
A

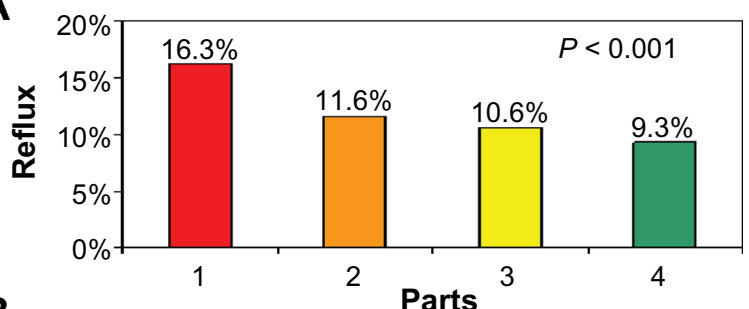

B

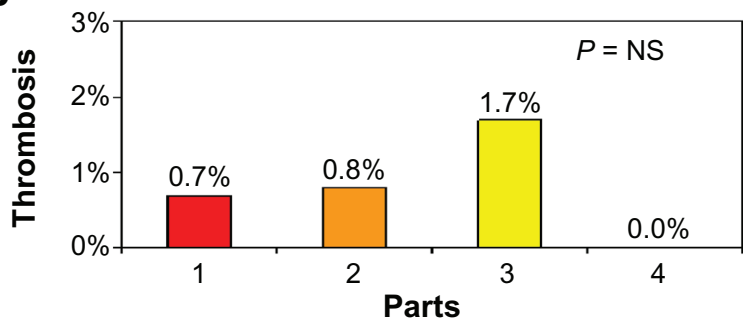

Figure 2 Prevalence of reflux (A) and thrombosis (B) by anatomical part.

of patients presenting with chronic venous disease, but it does not quantify the severity of this disorder. The noninvasive Doppler ultrasound procedure for diagnosing venous disease has been developed for the purposes of screening, damage assessment by verifying anatomic obstruction, and/or valve incompetence of the deep veins, superficial veins, and perforating veins. ${ }^{18}$ In fact, HD-ECD provides morphological information and enables reconstruction of the vascular anatomy and the diameter of blood vessels, allowing the creation of an accurate map for use during preoperative workup and the management of possible recurrences after surgery or sclerotherapy. Moreover, Doppler ultrasound can demonstrate reflux and quantify its severity, identify its origin, and follow the reflux axis craniodistally. The procedure has a higher difficulty rating in venous than in arterial disease, and thus requires greater operator experience.

The venous map, an anatomic paper pattern of the lower limbs venous system based on the ultrasound study, is an indispensable tool in the hands of phlebologists and can be

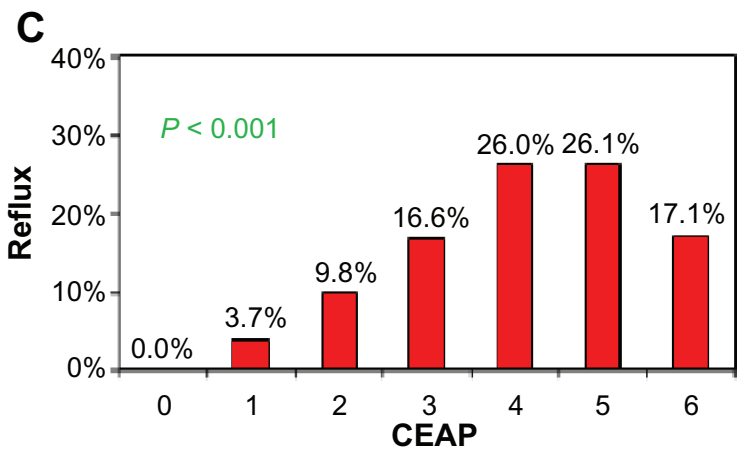

Figure 3 Association between reflux and CEAP stage. Abbreviation: CEAP, Clinical, Etiology, Anatomy, and Pathophysiology. considered the cornerstone of phlebology. In fact, Doppler ultrasound mapping is indispensable not only for diagnosis but also to guide the correct choice between conservative or demolitive therapy.

Our study proposes a venous mapping protocol for investigating the superficial and deep venous axes of the lower limbs, considering four anatomical parts (two for the thigh and two for the calf) using HD-ECD (see an example of the map in Figure 1). We also assessed interobserver variability in ultrasound evaluations, and the diagnostic accuracy of the imaging technique used to identify patients with venous diseases. Tracing few points in the overall venous tree of the lower limbs, we attempted to gain an easier picture of the anatomy of these vessels. The lack of a comparison with standard methods reduces the efficacy of our results and this could be considered a limitation of the study which needs to be developed further in the future.

Furthermore, the agreement between the two observers in detecting saphenectomy and thrombosis was perfect. Moreover, the agreement between the second versus the first operator was very high in detecting reflux (sensitivity 97.9, specificity 99.7, accuracy 99.5), underlining the fundamental role of this method in the morphofunctional study of the lower limb veins.

We observed a decreased prevalence of reflux in the 2nd, 3rd, and 4th parts as compared to the 1st anatomical part (Figure 2A). This result was in accordance with the concept of the blood column: in fact, an altered valve does not stop the blood column, which continues to backflow up to the next working valve that is able to stop the reflux. For this reason, reflux of the upper vein occurs earlier than reflux of the lower. Instead, thrombosis was not associated with the anatomical part (Figure 2B).

A study limitation could be considered the exclusion from the sample of patients suffering from peripheral artery diseases and/or cardiovascular disease. The choice of such an exclusion criteria which excludes many patients commonly considered in the angiology laboratory, was necessary in order to standardize our technique in a simpler way than obtained in the general population. A further step will involve the entire population in order to make the methods applicable to all the individuals requiring a venous system assessment.

In this study, we analyzed the role of echo color Doppler in the management of venous disease. We found that the higher CEAP classification stages were significantly associated with reflux $(P<0.001)$ (Figure 3$)$ as well as with thrombosis $(P<0.001)$. Thus, our findings show a strict association 
between the symptoms of venous disorders and the echo color Doppler results of four anatomical parts, with $99.5 \%$ accuracy. The relationship underlined in Figure 3 showed the relationship between each higher CEAP degree versus the lower ones (stage 0). Strangely, while observing 6th degree, we noticed less reflux percentages than 5 th or 4 th. Such a result seems quite unusual because, theoretically, the higher the CEAP degree, the more frequent the incidence of reflux. Maybe a patient selection bias could be considered, although a simple population variability could be an explanation of this issue.

In conclusion, this study demonstrated that our echo color Doppler evaluation protocol of the four anatomical parts of lower limb is a reliable method showing very low interobserver variability, which makes it accurate and reproducible for the assessment of the morphofunctional status of the lower limb veins.

\section{Conclusion}

This model for ultrasound venous mapping of the lower limbs, based on subdivision into four anatomical parts, shows very high interobserver agreement in the assessment of the lower limbs venous systems, and can be considered an alternative method for morphofunctional study of the lower limb veins to detect venous diseases. Strict observance of the protocol allows not only an accurate diagnosis of venous disease but also detection of the underlying causes, although novel studies are needed in order to compare such a new mapping method with standard protocols already used.

\section{Acknowledgments}

We would like to thank MV Pragnell for English text revision. We would like to thank Istituto di ricerche cliniche e biomediche "ricerca globale" Taranto Foundation for its help in chasing the aims of the study.

\section{Disclosure}

The authors report no conflicts of interest in this work.

\section{References}

1. Robertson L, Evans C, Fowkes FGR. Epidemiology of chronic venous disease. Phlebology. 2008;23:103-111.

2. Cruz CP, Eidt JF, Brown AT, Moursi M. Correlation between preoperative and postoperative duplex vein measurements of the greater saphenous vein used for infrainguinal arterial reconstruction. Vasc Endovascular Surg. 2004;38:57-62.

3. Zygmunt J Jr. What is new in duplex scanning of the venous system? Perspect Vasc Surg Endovasc Ther. 2009;21:94-104.

4. Nüllen H, Noppeney T. Diagnosis and treatment of varicose veins : part 2 : therapeutic procedures and results. Chirurg. 2010;81(12):1125-1138. German.

5. Hoballah JJ, Corry DC, Rossley N, Chalmers RT, Sharp WJ. Duplex saphenous vein mapping: venous occlusion and dependent position facilitate imaging. Vasc Endovascular Surg. 2002;36(5):377-380.

6. Davies AH, Magee TR, Jones DR, Hayward JK, Baird RN, Horrocks M. The value of duplex scanning with venous occlusion in the preoperative prediction of femoro-distal vein bypass graft diameter. Eur JVasc Surg. 1991;5(6):633-636.

7. Wong JK, Duncan JL, Nichols DM. Whole-leg duplex mapping for varicose veins: observations on patterns of reflux in recurrent and primary legs, with clinical correlation. Eur J Vasc Endovasc Surg. 2003;25(3): 267-275.

8. Cina A, Pedicelli A, Di Stasi C, et al. Color-Doppler sonography in chronic venous insufficiency: what the radiologist should know. Curr Probl Diagn Radiol. 2005;34(2):51-56.

9. Porter JM, Moneta GL. An international consensus committee on chronic venous disease. Reporting standards in venous disease (an update). J Vasc Surg. 1995;27:635-645.

10. Eklöf B, Rutherford RB, Bergan JJ, et al. Revision of the CEAP classification for chronic venous disorders (consensus statement). J Vasc Surg. 2004;40:1248-1252.

11. Zanatta N, Cioffi A, Brocco E, Mognol D, Santonastaso M. Appropriateness of requests of echo-color-Doppler tests. Recenti Prog Med. 2002;93(5):294-297. Italian.

12. Wittens CH, de Roos KP, van den Broek TA, et al. Guideline 'Diagnosis and treatment of varicose veins'. Ned Tijdschr Geneeskd. 2009;153:B71. Dutch.

13. Federici A, Ciccone M, Gattullo D, Losano G. Systolic and diastolic changes in human coronary blood flow during Valsalva manoeuvre. Clin Physiol. 2000;20(1):19-29.

14. Murgo JP, Westerhof N, Giolma JP, Altobelli SA. Manipulation of ascending aortic pressure and flow wave reflections with the Valsalva maneuver: relationship to input impedance. Circulation. 1981;63(1):122-132.

15. Labropoulos N, Tiongson J, Pryor L, et al. Definition of venous reflux in lower-extremity veins. J Vasc Surg. 2003;38(4):793-798.

16. Fowkes FGR, Evans CJ, Lee AJ. Prevalence and risk factors of chronic venous insufficiency. Angiology. 2001;52:S5-S15.

17. Bergan JJ, Schmid-Schonbein GW, Smith PDC, Nicolaides AN, Boisseau MR, Eklof B. Chronic venous disease. $N$ Engl J Med. 2006;355:488-498.

18. Krnić A, Vucić N, Sucić Z. Correlation of perforating vein incompetence with extent of great saphenous insufficiency: cross sectional study. Croat Med J. 2005;46(2):245-251.
Vascular Health and Risk Management

\section{Publish your work in this journal}

Vascular Health and Risk Management is an international, peerreviewed journal of therapeutics and risk management, focusing on concise rapid reporting of clinical studies on the processes involved in the maintenance of vascular health; the monitoring, prevention and treatment of vascular disease and its sequelae; and the involvement of

\section{Dovepress}

metabolic disorders, particularly diabetes. This journal is indexed on PubMed Central and MedLine. The manuscript management system is completely online and includes a very quick and fair peer-review system, which is all easy to use. Visit http://www.dovepress.com/ testimonials.php to read real quotes from published authors. 